\title{
Optical emission Spectral analysis of pulse modulated plasma
}

\author{
Deng longhu $^{1, a}$, Liu weifeng ${ }^{2, b}$, luyijia $^{3 .}$ c \\ 1,3Tsinghua Univ, Dept Mech Engn, Beijing 100084, Peoples R China. \\ ${ }^{2}$ China University of Geosciences, Dept Mech Engn,Beijing 10083, China. \\ adenglonghu001@163.com \\ bliuweifeng_2012@foxmail.com \\ cluyijia@tsinghua.edu.cn
}

\section{Key word: OES ; PMT ; high time resolution ; Pulse modulated plasma}

Abstract. Microelectronics manufacturing standards are increasingly demanding, plasma etching process is facing $22 \mathrm{~nm}$ or even smaller size of the node, which requires higher requirements on plasma processing technology. Pulse modulated plasma has been used since it has more advantages than traditional RF plasma. Pulse modulation plasma is difficult to measure due to the high frequency and the single pulse period is short, and optical emission spectroscopy (OES) is non-immersion test analysis means, in the plasma physics, atomic and molecular physics research has a wide range of applications. For non-constant states of the plasma, the emission spectrum also changes with time, and some of them change rapidly, such as pulse-modulated plasma, the current main photodetector ICCD camera and photoelectric multiplier tube (PMT) cannot meet the requirements, it is difficult to carry out effective measurement. In order to understand the complex physical and chemical processes in the plasma, a spectral analysis system based on PMT array was constructed based on the advantages of ICCD camera and photomultiplier tube. This paper mainly introduces the principle of spectrum analysis system based on PMT array, and uses this system to measure and analyze pulse modulation plasma.

\section{Introduction}

Optical emission spectroscopy is a non-immersion method, which is widely used in plasma physics, atomic and molecular physics and so on [1-3]. At present, the main spectral analysis of the photoelectric conversion equipment is ICCD camera and photomultiplier tube, ICCD camera can shoot a spectrum at the same time, despite the exposure time is very short, due to the accumulation of back-end charge, cannot achieve fast continuous shooting. Photomultiplier tubes have high temporal resolution and can reach the ns level, but the photomultiplier can only measure the spectral intensity of a single wavelength at the same time [4]. For non-constant states of the plasma, the emission spectrum also changes with time, and some of them change rapidly, the current main photodetector ICCD camera and photoelectric multiplier tube (PMT) cannot meet the requirements, it is difficult to carry out effective measurement. Based on the advantages and disadvantages of CCD cameras and photomultiplier tubes, the task force set up a spectral analysis system based on PMT. It can not only have high temporal resolution, but also can measure the light intensity at different wavelengths at the same time. The photomultiplier model used is the Hamamatsu Photon R928 and the header model is Hamamatsu Photon CC238. The structure of the spectrum analysis system based on PMT array is shown in Fig 1. The basic principle is the existence of a large number of excited particles in the plasma. When these excited states migrate from the excited state to the other excited states or return to the ground state, the excess energy will be emitted in the form of photons, thus forming the spectrum. These spectral information are closely related to the plasma state, and by detecting these spectral information, the parameters such as the temperature and density of the plasma can be analyzed. The physical diagram of the spectral analysis system based on the photomultiplier tube array is shown in Fig 2. 


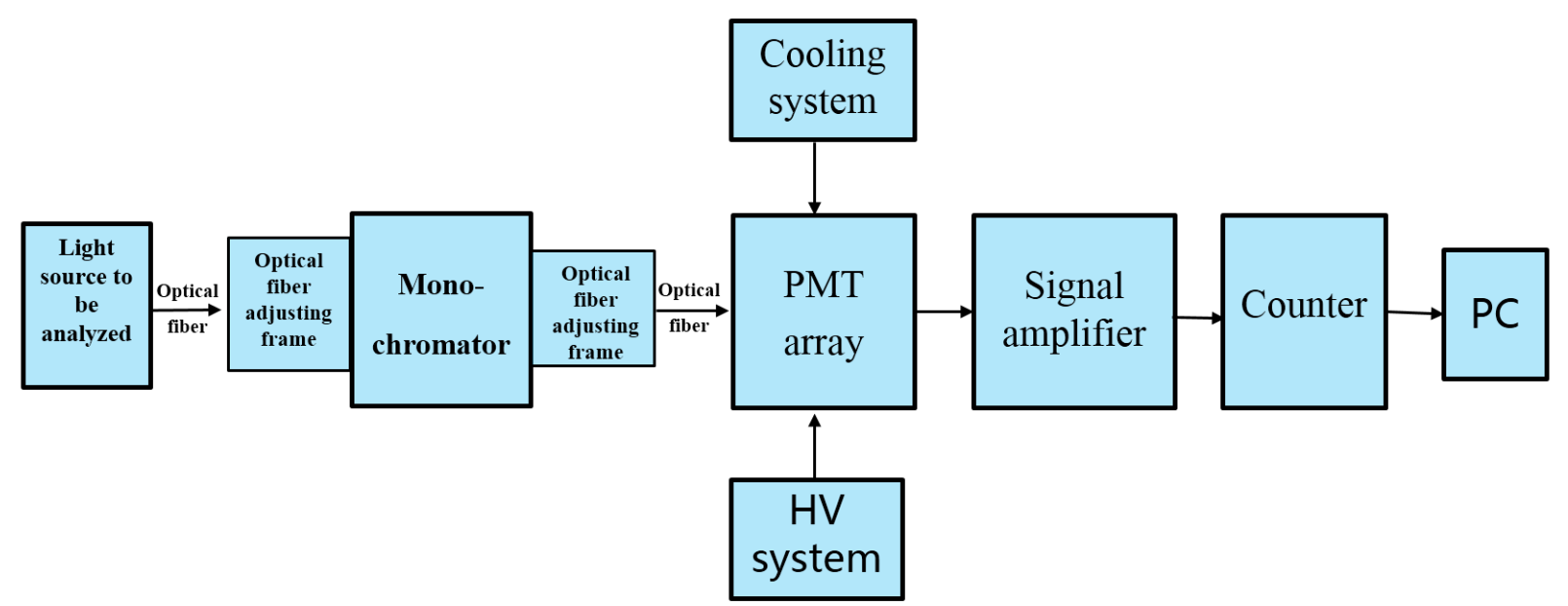

Fig. 1 The structure of the spectrum analysis system based on PMT array

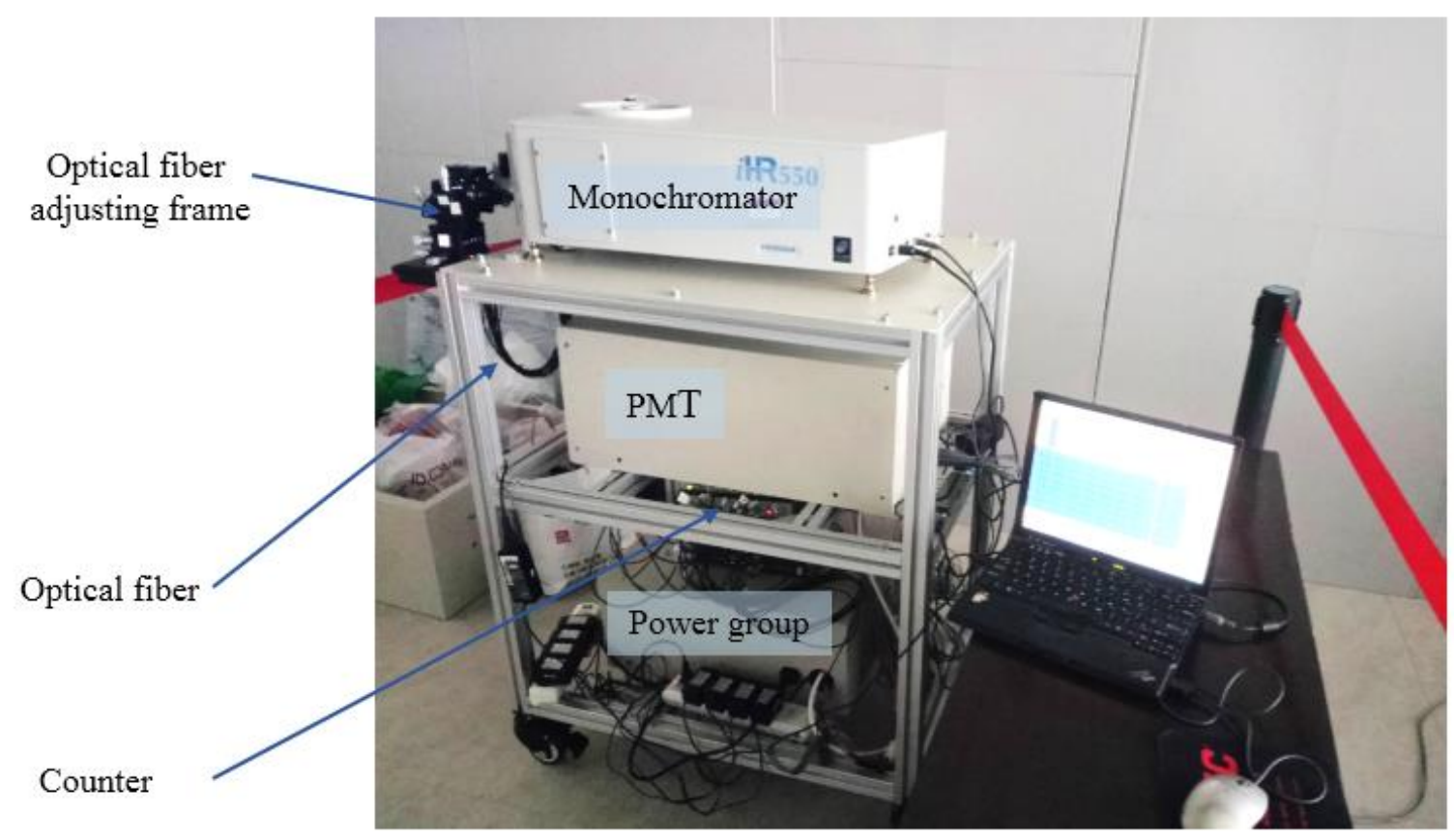

Fig. 2 The physical map of the spectrum analysis system based on PMT array

\section{Experiment}

The structure of the ICP chamber is shown in Figure 3[5], including the discharge chamber, matching network, gas supply, control system and so on. Discharge chamber radius $20 \mathrm{~cm}$, high $10 \mathrm{~cm}$, the experimental discharge power is $1 \mathrm{~kW}$.Ar gas pressure is $90 \mathrm{mtorr}$. The plasma glow passes through the focusing system located on the side wall of the vacuum chamber and enters the slit of the spectrum analysis system, and then is tested by the whole spectrum analysis system. 


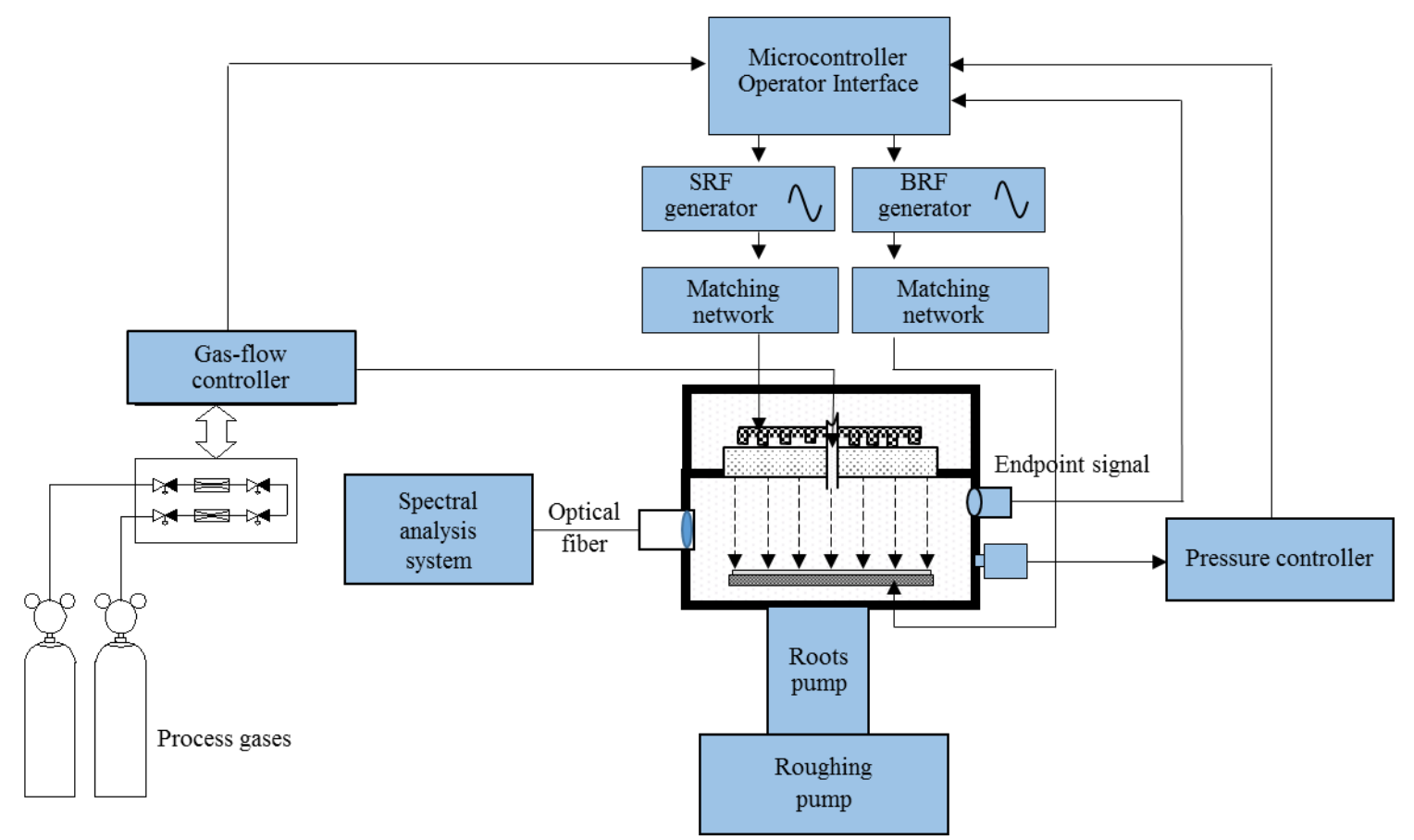

Fig.3 Discharge chamber structure

\section{Results and Analysis}

In the process of low pressure plasma discharge, Stark and natural broadening are very small and can be ignored. The instrument broadening size can be measured by the standard helium neon lamp. The broadening is mainly caused by Doppler broadening, and the size of the Doppler broadening [6] is

$$
\Delta \lambda_{D}=7.16 \times 10^{-7}\left(\frac{T_{E}}{M}\right)^{\frac{1}{2}} .
$$

The duty cycle of the pulse modulated plasma is $50 \%$ and $70 \%$, respectively. The test results are shown in figure 4. As can be seen from the results, the plasma temperature fluctuations are relatively large, generally high, may be due to the influence of the sheath, so that the measured temperature is too high.
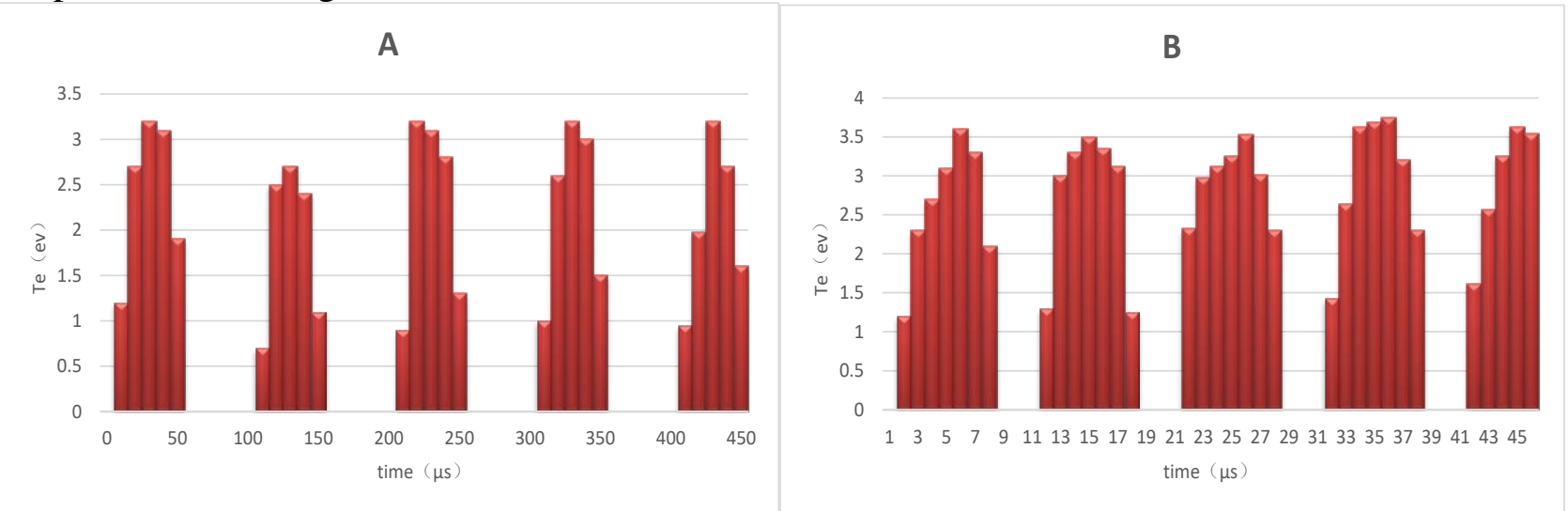

Fig.4 A duty cycle is $50 \%$ electron temperature B duty cycle is $70 \%$ electron temperature

In this paper, the structure and principle of the spectrum analysis system based on photomultiplier tube array are introduced. The system has high temporal resolution and can measure the light intensity at different wavelengths at the same time. The system is used to measure and analyze the pulse modulated plasma, and the results are analyzed. 


\section{References}

[1] Papageorgas P G, Winter $\mathrm{H}$, Albrecht $\mathrm{H}$, et al. A multichannel photon counting system for gas analysis with Raman-scattering technique [J]. IEEE Transactions on Instrumentation and Measurement, 1999, 48(6): 1166-1177.

[2] Xie Huiqiao, Tan Yi, Liu Yangqing, Wang Wenhao, Gao Zhe. Chinese joint collisional radiative model Spherical Tokamak helium plasma and its application in [J]. Journal of physics line intensity ratio diagnostic. 2014 (12)

[3] Wu Si Wang Quan Zu "modern physics experiment" (the selected experiment): Peking University press, 2001

[4] Brown R G W, Jones R, Rarity J G, et al. Characterization of silicon avalanche photodiodes for photon correlation measurements. 2: Active quenching [J]. Applied Optics, 1987, 26(12): 2383-2389.

[5] Zhang Yuan. Semiconductor manufacturing process [M]. Mechanical Industry Press, 2011

[6] Xiao X, Song L, Xiao W, et al. Space-dependent characterization of laser-induced plasma plume during fiber laser welding [J]. Journal of Physics D: Applied Physics, 2016, 49(48): 485203. 